\title{
Parameter Estimation of Single Phase Core Type Transformer Using Bacterial Foraging Algorithm
}

\author{
Seeni Padma*, Srikrishna Subramanian \\ Department of Electrical Engineering, Faculty of Engineering and Technology, Annamalai University, \\ Annamalainagar, India \\ E-mail:padma_pnr@yahoo.co.in,profdrmani@gmail.com \\ Received June 9, 2010; revised September 6, 2010; accepted October 14, 2010
}

\begin{abstract}
The electrical circuit equivalents of magnetic device structures such as transformer require an exact knowledge of its parameters. Efficient parameter estimation technique is essential to obtain the equivalent circuit parameters of transformer because the parameters are used to manipulate parasitic elements and to obtain the enhanced circuit performance. In this paper, Bacterial Foraging Algorithm (BFA) has been applied to estimate the equivalent circuit parameters of single phase core type transformer. The information of open Circuit (OC) and Short Circuit (SC) tests has been utilized in BFA algorithm. The effectiveness of the proposed approach has been tested with a sample transformer and the simulation results are compared against the conventional method. The numerical results show that the proposed approach outperforms the conventional method in the aspects of solution quality.
\end{abstract}

Keywords: Parameter Estimation, Transformer Equivalent Circuit, Bacterial Foraging Algorithm

\section{Introduction}

Transformer is one of the most common nonlinear elements in power systems. The nonlinearity is caused by the magnetizing characteristics of the transformer iron core. Particularly, single phase transformers are often used in power electronic circuit and power system applications. Estimation of the equivalent circuit parameters is useful for circuit performance analysis, transient studies, identification of parasitic elements etc. The electromagnetic transient studies require an adequate modeling of the transformer equivalent circuit and various techniques have been applied for parameter estimation. The ferroresonance of the transformer has been predicted or confirmed and its severity can be evaluated by using transformer equivalent circuit models [1]. A topology-based and duality derived three-phase three winding core type transformer model has been developed and it treats the leakage inductances and the coupling effects of the core in a straightforward and integrated way [2]. The method based on Genetic Algorithm (GA) has been developed for the identification of synchronous machine parameters from short circuit tests [3].

GA has also been applied to determine the electric pa- rameters of an induction machine using Park model [4]. The average winding temperature rise under its field operation conditions and rise in winding temperature has been determined from the estimated values of winding resistance [5]. The Park model electric parameters of an induction machine [6] are used in control techniques for variable speed drives, have been estimated by GA. The parameters of a saturation model of transformer are also estimated by using the data from transformer inrush tests and steady state operation [7].

An alternative approach to conventional open and short-circuit test for determining the parameters of $\mathrm{N}$-windings transformer operating at power frequency on an on-line mode. The method is based on linear Least Error Square (LES) algorithm and uses the digitized samples of the input current and voltage as well as the output current and voltage of the transformer windings [8]. The GA based method has also been suggested to identify the parameters of an induction motor [9].

The conventional model for multiwinding transformers is difficult to relate to its physical construction, and the measurement of the model parameters is also difficult and unreliable, [10] hence a physically based electrical model of a high voltage multiwinding transformers has 
been developed. In this model, each component corresponds to a physical quantity of the transformer and the leakage inductance for nonuniformly spaced windings, which store significant energy in the flux in the radial field, has also been easily calculated.

Differential evolution algorithm [11] has been applied for parameter identification of an induction motor. Parameter identification of an induction machine using GA [12] has been discussed for variable speed applications. The general mathematical model of the motor based upon Kron's voltage equations has been considered to estimate the parameters and the start-up performance of the motor has been used as the measurement for identification process. A multi-stage transformer model [13] for high frequency transient operation is established, and the equivalent circuit parameters are estimated by using their mathematical formulation. The modified version of GA namely, enhanced GA [14] which operates on real-valued parameter sets and provides an improvement in the solution quality, has been applied to determine the equivalent circuit parameters of induction motors. The modern heuristic search technique, called Bacterial Foraging Algorithm (BFA) has been developed based on modelling of bacteria E.coli behavior present in human intestine and it has been proven that is efficient [15-19] for various engineering optimization problems. In this article, BFA has been applied to estimate the equivalent circuit parameters of single phase core type transformer. The effectiveness of the proposed BFA approach has been tested with the suitable transformer.

\section{Problem Description and Approach for Solution}

The equivalent circuit model of a Single phase transformer is shown in Figure 1. The conventional method requires a minimum of two tests namely short circuit test and open circuit test to determine the equivalent circuit parameters. The performance evaluation of transformer using the equivalent circuit parameters is inaccurate because the change in the winding resistance due to temperature rise which is caused by loading effect. For the above reason, the exact equivalent circuit model of transformer has been formulated by including the load impedance as shown in Figure 2.

The estimation of equivalent circuit parameters with good accuracy is important as they have direct influence in the performance computations such as efficiency, voltage regulation, etc. The parameter estimation of transformer depends on both of its physical features and actual operating conditions hence a powerful tool is required to estimate the transformer equivalent parameters.

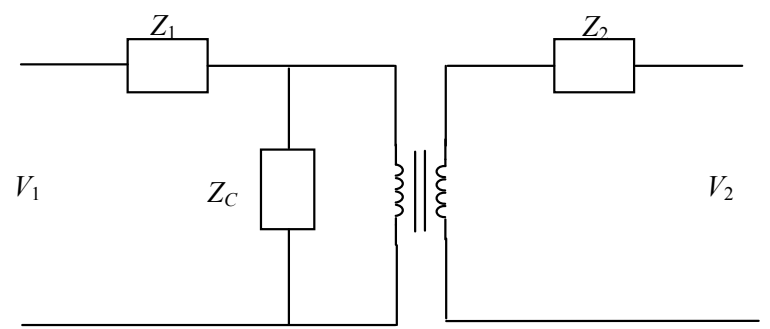

Figure 1. Equivalent circuit model of a single phase of transformer.

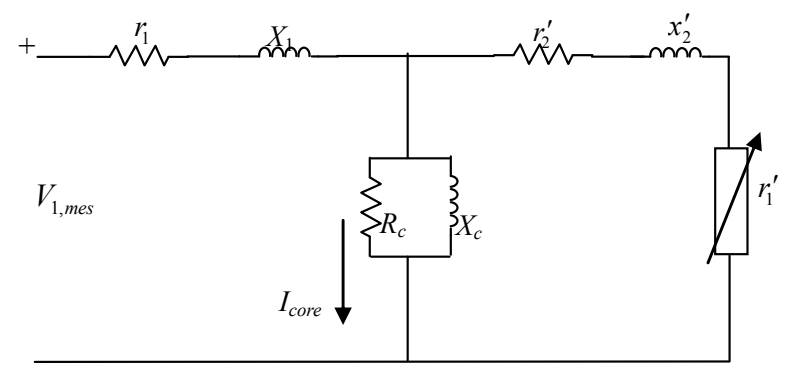

Figure 2. Exact equivalent circuit model of a single phase transformer.

The modern heuristic search technique, bacterial foraging algorithm has been used to determine the equivalent circuit parameters.

The necessary objective function to be used for parameter estimation is obtained as follows. Let the impedance of the primary and secondary windings referred to primary side are

$$
\begin{gathered}
z_{1}=\sqrt{r_{1}^{2}+x_{1}^{2}} \\
z_{2}=\sqrt{\left(r_{2}^{\prime}+r_{1}^{\prime}\right)^{2}+\left(x_{2}^{\prime}\right)^{2}}
\end{gathered}
$$

Then the admittance of the magnetizing winding is

$$
Y_{c}=\frac{1}{R_{c}}-\frac{1}{X_{c}}
$$

Therefore, the impedance of magnetizing winding is

$$
Z_{C}=\frac{1}{Y_{C}}
$$

The estimated value of the equivalent impedance as referred to primary side is

$$
Z_{\text {lest }}=Z_{1}+\left(Z_{2}^{\prime} Z_{C} /\left(Z_{2}^{\prime}+Z_{C}\right)\right)
$$

The estimated value of primary voltage be

$$
V_{\text {lest }}=Z_{1 e s t} I_{1}
$$

And the estimated value of input power be

$$
P_{\text {lest }}=P_{\text {core }}+P_{c u}+P_{2}
$$

The objective of the parameter problem is to find a set of equivalent circuit parameters that minimizes the error. The equivalent circuit parameters are to be estimated by 
minimizing the following objective function,

$$
f(X)=f_{1}^{2}+f_{2}^{2}
$$

where $X=r_{1}, x_{1}, r_{2}^{\prime}, x_{2}^{\prime}, R_{c}, X_{c}$

$$
\begin{aligned}
& f_{1}=\left(\left(v_{1 \text { mes }}-v_{\text {lest }}\right) / v_{1 \text { mes }}\right) * 100 \\
& f_{2}=\left(\left(P_{1 \text { mes }}-P_{\text {lest }}\right) / P_{1 \text { mes }}\right) * 100
\end{aligned}
$$

\section{Bacterial Foraging Optimization Techniques}

Natural selection tends to eliminate animals with poor foraging strategies and favour the propagation of genes of those animals that have successful foraging strategies since they are more likely to enjoy reproductive success. After many generations, poor foraging strategies are either eliminated or shaped into good ones. The E. coli bacteria that are present in our intestines also undergo a foraging strategy. The control system of these bacteria that dictates how foraging should proceed can be subdivided into four sections namely Chemotaxis, Swarming, Reproduction and Elimination and Dispersal.

1) Chemotaxis: This process is achieved through swimming and tumbling via Flagella. Depending upon the rotation of Flagella in each bacterium, it decides whether it should move in a predefined direction (swimming) or altogether in different directions (tumbling), in the entire lifetime. To represent a tumble, a unit length random direction, say $\phi(j)$, is generated; this will be used to define the direction of movement after a tumble.

In particular

$$
\theta^{i}(j+1, k, 1)=\theta^{i}(j, k, 1)+C(i) \varphi(j)
$$

where $\theta^{i}(j, k, l)$ represents the $i$-th bacterium at $j$-th chemotactic, $k$-th reproductive and $i$-th elimination and dispersal step. $C(i)$ is the size of the step taken in the random direction specified by the tumble (run length unit).

2) Swarming: During the process of reaching towards the best food location it is always desired that the bacterium which has searched the optimum path should try to provide an attraction signal to other bacteria so that they swarm together to reach the desired location. In this process, the bacteria congregate into groups and hence move as concentric patterns of groups with high bacterial density.

The mathematical representation for swarming can be represented by

$$
\begin{aligned}
& J_{c c}(\theta, P(j, k, 1))=\sum_{i=1}^{s} J_{c c}^{i}\left(\theta, \theta^{i}(j, k, 1)\right) \\
& =\sum_{i=1}^{s}\left[-d_{\text {attract }} \exp \left[-W_{\text {attract }} \sum_{m=1}^{p}\left(\theta_{m}-\theta_{m}^{i}\right)^{2}\right]\right]+ \\
& \sum_{i=1}^{s}\left[h_{\text {repelent }} \exp \left[-W_{\text {repelent }} \sum_{m=1}^{p}\left(\theta_{m}-\theta_{m}^{i}\right)^{2}\right]\right]
\end{aligned}
$$

where $J_{c c}(\theta, P(j, k, l))$ is the cost function value to be added to the actual cost function to be minimized to present a time varying cost function. " $S$ " is the total number of bacteria and " $p$ " the number of parameters to be optimized which are present in each bacterium. $d_{\text {atract }}, w_{\text {attract }}$, $h_{\text {repellent, }} w_{\text {repellent }}$ are different coefficients that are to be chosen properly.

3) Reproduction: The least healthy bacteria die and the other healthiest bacteria each split into two bacteria, which are placed in the same location. This makes the population of bacteria constant.

4) Elimination and Dispersal: It is possible that in the local environment the live of a population of bacteria changes either gradually (e.g., via consumption of nutrients) or suddenly due to some other influence. Events can occur such that all the bacteria in a region are killed or a group is dispersed into a new part of the environment. They have the effect of possibly destroying the chemotactic progress, but they also have the effect of assisting in chemotaxis, since dispersal may place bacteria near good food sources. From a broad perspective, elimination and dispersal are parts of the population-level long-distance motile behavior. The proposed method is summarized by the flow chart shown in Figure 3.

\section{Bacterial Foraging Algorithm Based Parameter Estimation}

In this paper the bacterial foraging algorithm has been used for search the optimal transformer equivalent circuit parameters. Each bacterium $X$ contains six members namely $r_{1}, x_{1}, r_{2}^{\prime}, \quad x_{2}^{\prime}, R_{c}$ and $X_{c}$. The process of estimate the equivalent circuit parameters of the transformer can be explained as follows: First input the bacterial foraging parameters and conventional measured data, and also specify lower and upper limits of the equivalent circuit parameters. Generate the positions of the equivalent circuit parameter randomly and evaluate the objective value of each bacterium. After evaluating the objective function, modify the position of the equivalent circuit parameters for all the bacteria using the tumbling/ swimming process and perform reproduction and elimination operation. The output of equivalent circuit parameter is obtained when the maximum steps is reached. Finally, compute the operating performances of the transformer such as efficiency and regulation. In proposed method, the process of "chemotaxis" enables bacteria to obtain a satisfactory ability of local search. It is worth notice that the individuals in bacterial foraging algorithm could converge rapidly without information sharing between each other, which is different from other methods.

Parameter estimation procedure is performed in ac- 


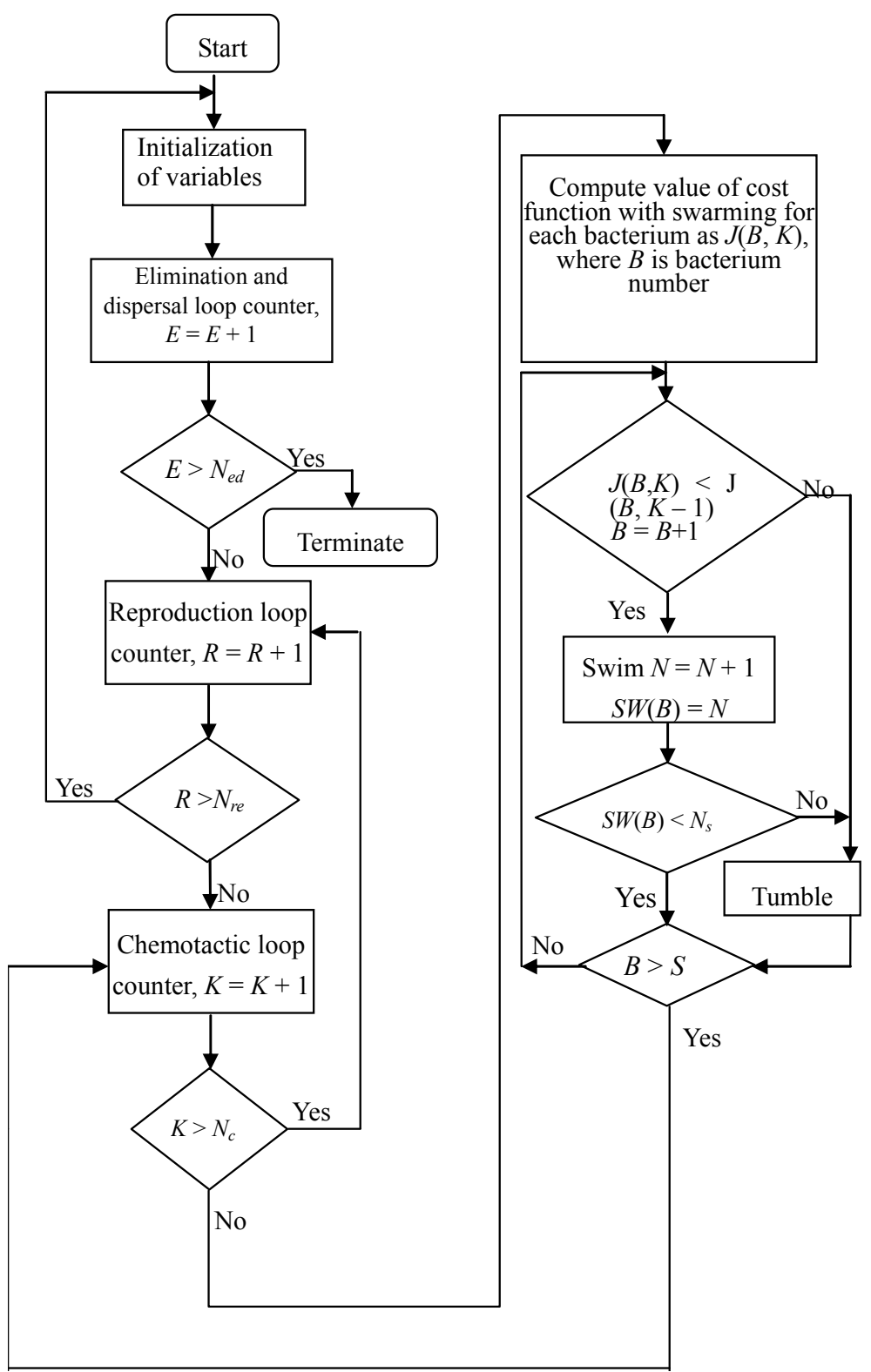

Figure 3. Flowchart of bacterial foraging algorithm.

cordance with the following steps.

Step-1 Initialize parameters $P, s, N_{r e}, N_{e d}, p_{e d}, C(i)(i=$ $1,2, \cdots, s)$, and $X_{i}$. Also initialize all the counter values to zero.

Step-2 Elimination-dispersal loop: $l=l+1$

Step-3 Reproduction loop: $k=k+1$

Step-4 Chemotaxis loop: $j=j+1$

1) For $i=1,2, \cdots, S$, calculate cost function value and efficiency- for each bacterium $i$ as follows.

a) $N_{i s}$ signal samples are passed through the model.

b) The output is then compared with the corresponding desired signal to calculate the error.

c) The same of the squared error averaged over $N_{i s}$ is finally stored in $J(i, j, k, l)$. The cost function is calculated for number of input samples.

d) End of for loop.

2) For $i=1,2, \cdots, S$ take the tumbling/swimming decision

Tumble: Generate a random vector $\Delta(i)$ with each element

$\Delta_{m}(i) m=1,2, p, a$ random number.

Move: Let

$$
\theta^{i}(j+1, k, 1)=\theta^{i}(j, k, 1)+C(i) \frac{\Delta(i)}{\sqrt{\Delta^{T}(i) \Delta(i)}}
$$

Fixed step size in the direction of tumble for bacterium 
$i$ is considered.

Compute $J(i, j+1, k, l)$ and then

Let

$J_{s w}(i, j+1, k, 1)=J(i, j+1, k, 1)+J_{c c}\left(\theta^{i}(j+1, k, 1), P(j+1, k, 1)\right)$

Swim:

i) Let $m=0$; (counter for swim length)

ii) While $m<N_{s}$ (have not climbed down too long)

- Let $m=m+1$

- $\quad$ If $J_{s w}(i, j+1, k, l)<J_{\text {last }}$ (if doing better), let $J_{\text {last }}=$ $J_{S w}(i, j+1, k, l)$ and Let

$$
\theta^{i}(j+l, k, l)=\theta^{i}(j, k, l)+C(i) \frac{\Delta(i)}{\sqrt{\Delta^{T}(i) \Delta(i)}} \text { and use }
$$

this $\theta^{i}(j+1, k, l)$ to compute the new $J(i, j+1, k, l)$

- $\quad$ Else, let $m=N_{s}$. This is the end of the while statement.

3) Go to next bacterium $(i+1)$ if $i \neq S$ (i.e. go to $b$ ) to process the next bacterium.

Step-5 If $j<N_{c}$, go to step 4. In this case, continue chemotaxis since the life of the bacteria is not over.

Step-6 Reproduction:

a) For the given $k$ and $l$, and for each $i=1,2, \cdots, s$,

Let $J_{\text {health }}^{i}=\min J_{S w}(i, j, k, l)$ be the health of the bacterium $i$ (a measure of how many nutrients it got over its life time and how successful it was at avoiding noxious substance). Sort bacteria in order of ascending cost $J_{\text {health }}$ (higher cost means lower health).

b) The $S_{r}=s / 2$ bacteria with highest $J_{\text {health }}$ values die and other $S_{r}$ bacteria with the best value split (and the copies that are made are placed at the same location as their parent).

Step-7 If $k<N_{\text {re }}$ go to 3 . In this case, the number of specified reproduction steps has not been reached, so the next generation of the chemotactic loop is started.

Step-8 Elimination-dispersal: For $i=1,2, \cdots, s$, with probability $P_{e d}$, eliminates and disperses each bacterium (this keeps the number of bacteria in the population constant). To do this, if a bacterium is eliminated, simply disperse another one to a random location on the optimization domain. If $l<N_{e d}$, then go to step 2; otherwise, print the results and stop.

\section{Simulation Results and Discussion}

To verify the effectiveness of proposed parameter estimation technique, the algorithm is used to estimate the parameters of a single phase, two-winding, core type transformer and its specifications are presented in Table 1. By conducting the load test, the performance of transformer at various loading conditions is obtained and is presented in Table 2. The results of proposed method are compared with the load test results.

The equivalent circuit parameters are also determined by performing the suitable tests and the predetermined
Table 1. Specification of sample transformer.

\begin{tabular}{cc}
\hline \multicolumn{2}{c}{ Specifications } \\
\hline Capacity & $2 \mathrm{KVA}$ \\
Primary voltage & $230 \mathrm{~V}$ \\
Secondary voltage & $115 \mathrm{~V}$ \\
Frequency & $50 \mathrm{~Hz}$ \\
\hline
\end{tabular}

efficiency and voltage regulation are compared with the load test values which are presented in Table 3. From the comparison, it is clear that there is a deviation in the values of efficiency and regulation.

By estimating the accurate value of equivalent circuit parameters, the deviation can be minimized. Hence the problem has been formulated as an error minimization problem and the modern heuristic search technique, BFA has been applied to obtain the optimal value of equivalent circuit parameters. The parameters of the BFA used for the simulation studies are summarized in Table 4.

The optimal value of equivalent circuit parameters of aforementioned single phase transformer are obtained by applying BFA. The best results are obtained from 20 trail runs and are detailed in Table 5. For the sake of comparison, rated load condition is considered and the efficiency and voltage regulation at rated load are predetermined by using the estimated equivalent circuit parameters. The simulation results obtained by the proposed method and actual load test measurement are compared in Table 5. From the comparison, it is revealed that an improvement in the predetermined efficiency and voltage regulation.

The simulation studies have been extended to various load conditions. For each load conditions, the efficiency and voltage regulation are predetermined. The obtained results at various load conditions are compared with the load test measurements and the comparison is presented in Table 6. In addition, the percentage of error over the load test measurement is computed and is also presented in Table 6. The comparison clearly shows the reduction in error between the actual and estimated data. The equivalent circuit parameters obtained by the proposed methodology has close agreement with the conventional method. In all load conditions, a slight increase in efficiency and a comparable reduction in voltage regulation have been achieved.

The performance curve of efficiency and regulation obtained from conventional and proposed method are shown in Figures $\mathbf{4}$ and $\mathbf{5}$ respectively. It is obvious that the performance characteristics of the sample transformer using BFA based parameter estimation method shows the better performance than conventional method.

The convergence characteristic of the proposed method for a sample transformer is plotted in Figure 6. It shows that proposed method converges very quickly hence, it has a good convergence property which confirms that 
Table 2. Load test data of a single phase transformer at $230 \mathrm{~V}, 50 \mathrm{~Hz}$ supply.

\begin{tabular}{ccccccccc}
\hline Load \% & $\mathrm{V}_{1}(\mathrm{~V})$ & $\mathrm{V}_{2}(\mathrm{~V})$ & $\mathrm{I}_{1}(\mathrm{~A})$ & $\mathrm{I}_{2}(\mathrm{~A})$ & $\mathrm{P}_{1}(\mathrm{~W})$ & $\mathrm{P}_{2}(\mathrm{~W})$ & Efficiency (\%) & Regulation (\%) \\
\hline 50 & 226 & 109 & 4.6 & 8.7 & 1000 & 948.3 & 94.83 & 4.385 \\
60 & 225 & 108 & 5.4 & 10.4 & 1180 & 1123.2 & 95.19 & 5.263 \\
70 & 225 & 108 & 6.3 & 12.2 & 1400 & 1317.6 & 94.11 & 5.268 \\
80 & 223 & 107 & 7.1 & 13.9 & 1568 & 1487.3 & 94.85 & 6.140 \\
90 & 223 & 106 & 8.0 & 15.7 & 1768 & 1664.2 & 94.13 & 7.017 \\
100 & 223 & 105 & 8.7 & 17.39 & 1940 & 1826 & 94.12 & 7.894 \\
\hline
\end{tabular}

Table 3. Directly measured parameters and performance.

\begin{tabular}{ccccccccc}
\hline Parameters & $\boldsymbol{r}_{\mathbf{1}}$ & $\boldsymbol{x}_{\mathbf{1}}$ & $\boldsymbol{R}_{\mathbf{2}}^{\prime}$ & $\boldsymbol{x}_{\mathbf{2}}^{\prime}$ & $\boldsymbol{R}_{\boldsymbol{c}}$ & $\boldsymbol{X}_{\boldsymbol{c}}$ & Efficiency (\%) & Regulation (\%) \\
\hline Measured $^{\mathrm{a}}$ & 0.428 & 0.21 & 0.508 & 0.03 & 1437.5 & 294.8 & 94.6 & 3.765 \\
Measured $^{\mathrm{b}}$ & - & - & - & - & - & - & 94.12 & 7.894 \\
\hline
\end{tabular}

a: Parameters measured from OC and SC tests; b: Full load performance directly measured from load test.

Table 4. Parameter used for BFA method.

\begin{tabular}{cc}
\hline Parameter & Value \\
\hline Number of bacterium $(s)$ & 20 \\
Number of chemotatic steps $\left(N_{c}\right)$ & 10 \\
Swimming length $\left(N_{s}\right)$ & 4 \\
Number of reproduction steps $\left(N_{r e}\right)$ & 4 \\
Number of elimination and dispersal events $\left(N_{\text {ed }}\right)$ & 5 \\
Depth of attractant $\left(d_{\text {attract }}\right)$ & 0.1 \\
Width of attractant $\left(\omega_{\text {attract }}\right)$ & 0.2 \\
Height of repellent $\left(h_{\text {repellant }}\right)$ & 0.1 \\
Width of repellent $\left(\omega_{\text {repellant }}\right)$ & 10 \\
Probability of elimination-dispersal events $\left(P_{\text {ed }}\right)$ & 0.02 \\
\hline
\end{tabular}

Table 5. Estimated parameters of single phase transformer using BFA.

\begin{tabular}{ccccccccc}
\hline Parameters & $\boldsymbol{r}_{\mathbf{1}}$ & $\boldsymbol{x}_{\mathbf{1}}$ & $\boldsymbol{r}_{\mathbf{2}}^{\prime}$ & $\boldsymbol{x}_{\mathbf{2}}^{\prime}$ & $\boldsymbol{R}_{\boldsymbol{c}}$ & $\boldsymbol{X}_{\boldsymbol{c}}$ & Efficiency (\%) & Regulation (\%) \\
\hline Estimated & 0.428 & 0.43 & 0.493 & 0.024 & 1437.5 & 294.226 & 94.23 & 4.27 \\
Measured & - & - & - & - & - & - & 94.12 & 7.894 \\
\hline
\end{tabular}

Table 6. BFA based estimated parameters at various load points.

\begin{tabular}{ccccccc}
\hline \multirow{2}{*}{$\begin{array}{c}\text { Percentage } \\
\text { load }\end{array}$} & \multicolumn{3}{c}{ Efficiency } & \multicolumn{3}{c}{ Voltage regulation } \\
\cline { 2 - 6 } & Measured & BFA results & \% Error & Measured & BFA results & \% Error \\
\hline 50 & 94.83 & 94.92 & 0.09 & 4.385 & 2.1317 & -2.25 \\
60 & 95.19 & 95.32 & 0.13 & 5.263 & 2.554 & -2.70 \\
70 & 94.11 & 94.50 & 0.39 & 5.268 & 2.996 & -2.27 \\
80 & 94.85 & 94.89 & 0.04 & 6.140 & 3.414 & -2.72 \\
90 & 94.13 & 94.30 & 0.17 & 7.017 & 3.856 & -3.16 \\
100 & 94.12 & 94.23 & 0.11 & 7.894 & 4.271 & -3.62 \\
\hline
\end{tabular}

the BFA technique is suitable for determining the global optimum solution. The simulation study clearly shows that the proposed method is efficient for parameter estimation of transformer.

\section{Conclusions}

The parameter estimation of single phase core type transformer using bacterial foraging algorithm has been presented. The performance characteristics, such as efficiency and voltage regulation are considered along with the name plate data in order to minimize the error between the estimated and measured data. The feasibility of the proposed method has been validated with a single phase transformer. The simulation results obtained by the proposed method are compared with the equivalent cir- 


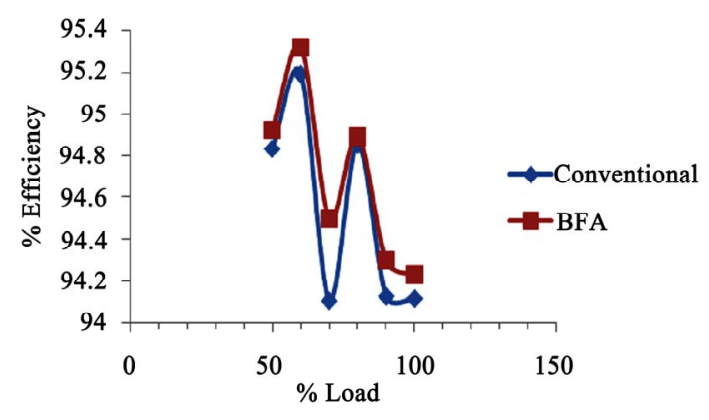

Figure 4. Performance curve of efficiency.

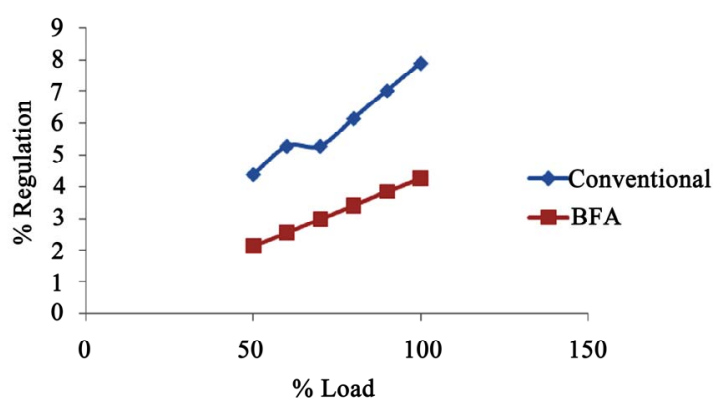

Figure 5. Performance curve of regulation.

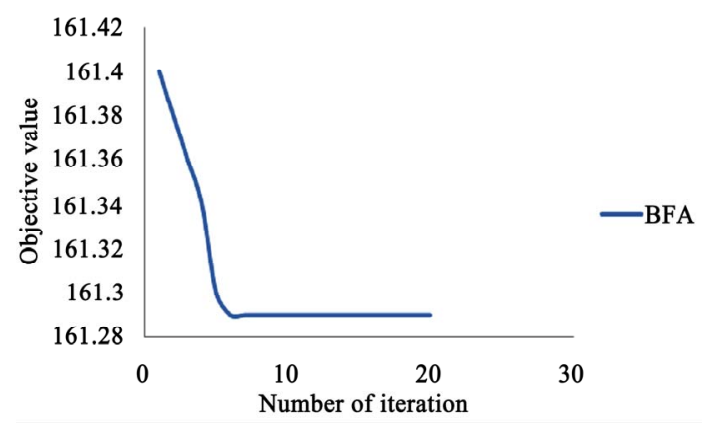

Figure 6. Convergence curve.

cuit method and the load test. The results show that the proposed method provides lesser error than the equivalent circuit method, and has good solution quality and convergence behavior. The proposed method can be applied to any capacity of transformer. The BFA technique promises to be quite efficient in solving highly nonlinear optimization problem so its application in some other fields may also be tried.

\section{Acknowledgements}

The authors are grateful to the authorities of Annamalai University for providing all facilities to carry out this work.

\section{References}

[1] B. A. Mork, "Five-Legged Wound-Core Transformer Model:
Derivation, Parameters, Implementation, and Evaluation," IEEE Transactions on Power Delivery, Vol. 14, No. 4, October 1999, pp. 1519-1525.

[2] X. S. Chen and S. S. Venkata, "A Three-Phase ThreeWinding Core-Type Transformer Model for Low-Frequency Transient Studies," IEEE Transactions on Power Delivery, Vol. 12, No. 2, April 1997, pp. 775-781.

[3] H. K. M. Youssef and K. M. Naggar, "Genetic-Based Algorithm for Identification Synchronous Machine Parameter Using Short Circuit Tests," International Journal of Energy Research, Vol. 24, No. 10, 2000, pp. 877-885.

[4] P. Pillay, V. Levin, P. Otaduy and J. Kueck, "In-Situ Induction Motor Efficiency Determination Using the Genetic Algorithm," IEEE Transactions on Energy Conversion, Vol. 13, No. 4, December 1998, pp. 326-333.

[5] S. H. Thilagar and G. S. Rao, "In-Situ Determination of Transformer Winding Temperature Rise Using Genetic Algorithm Based Parameter Estimation," Proceedings of 2nd International Conference on Electrical and Computer Engineering, Dhaka, 26-28 December 2002.

[6] B. Abdelhadi, A. Benoudijit and N. Nait-Said, "Application of Genetic Algorithm with a Novel Adaptive Scheme for the Identification of Induction Machine Parameters," IEEE Transactions on Energy conversion, Vol. 20, No. 2, June 2005, pp. 284-291.

[7] B. L. Peacock, M. Steurer, J. Langston, T. Baldwin and S. Henry, "Estimation of Parameters for Digital Modeling of Saturation Effects for a Transformer Model for a RealTime Simulator," 41st Southeastern Symposium on System Theory, Tullahoma, 15-17 March 2009, pp. 306-310.

[8] S. A. Soliman, R. A. Alammari and M. A. Mostafa, "OnLine Estimation of Transformer Model Parameters," Proceedings of Large Engineering System Conference on Power Engineering, Halifax, 28-30 July 2004, pp. 170178.

[9] F. Alonge, F. D'lppolito, G. Ferrante and F. M. Raimondi, "Parameter Identification of Induction Motor Model Using Genetic Algorithms," IEE Proceedings of Control Theory Applications, Vol. 145, No. 6, November 1998, pp. 587-597.

[10] J. Wang, A. F. Witulski, J. L. Vollin, T. K. Phelps and G. I. Cardwell, "Derivation, Calculation and Measurement of Parameters for a Multi-Winding Transformer Electrical Model," Proceedings of IEEE Applied Power Electronics Conference, Dallas, Vol. 1, 14-18 March 1999, pp. 220-226.

[11] R. K. Ursem and P. Vadstrup, "Parameter Identification of Induction Motors Using Differential Evolution," Proceedings of the Congress on Evolutionary Computation, Canberra, Vol. 2, 8-12 December 2003, pp. 790-796.

[12] K. S. Huang, W. Kent, Q. H. Wu and D. R. Turner, "Parameter Identification of an Induction Machine Using Genetic Algorithms," International Symposium on Computer Aided Control System Design, Hawaii, 22-27 August 1999, pp. 510-515.

[13] A. Keyhani, A. Tsai and S. Sebo, "Modeling and Parameter Estimation of Power Transformers for the Study of High Frequency System Transients," Proceedings of 
the 32nd Midwest Symposium on Circuits and Systems, Urbana, Vol. 1, 14-16 August 1989, pp. 258-264.

[14] P. Y. Chung, M. Dölen and R. D. Lorenz, "Parameter Identification for Induction Machines by Continuous Genetic Algorithms," Proceedings of Annual Neural Network in Engineering Conference, St. Louis, 5-8 November 2000, pp. 1-13.

[15] W. J Tang, Q. H. Wu and J. R. Saunders, "Bacterial Foraging Algorithm for Dynamic Environments," Proceedings of IEEE Congress on Evolutionary Computation, Vancouver, 16-21 July 2006, pp. 1324-1330.

[16] D. P. Acharya, G. Panda, S. Mishra and Y. V. S. Lakshmi, "Bacteria Foraging Based Independent Component Analysis," Proceedings of International Conference on Computational Intelligence and Multimedia Applications, Si- vakasi, 13-15 December 2007, pp. 527-531.

[17] B. K. Panigrahi and V. R. Pandi, "Bacterial Foraging Optimisation: Nelder-Mead Hybrid Algorithm for Economic Load Dispatch," IET Generation, Transmission and Distribution, Vol. 2, No. 4, 2008, pp. 556-565.

[18] M. Tripathy and S. Mishra, "Bacteria Foraging Based Solution to Optimize Both Real Power Loss and Voltage Stability Limit," IEEE Transactions on Power Systems, Vol. 22, No. 1, February 2007, pp. 240-248.

[19] G. Noriega, J. Restrepo, V. Guzmán, M. Giménez and J. Aller, "On Line Parameter Estimation of Electric Systems Using the Bacterial Foraging Algorithm," Proceedings of 13th European Conference on Power Electronics and Applications, Barcelona, 8-10 September 2009, pp. 1-7. 


\section{Nomenclature}

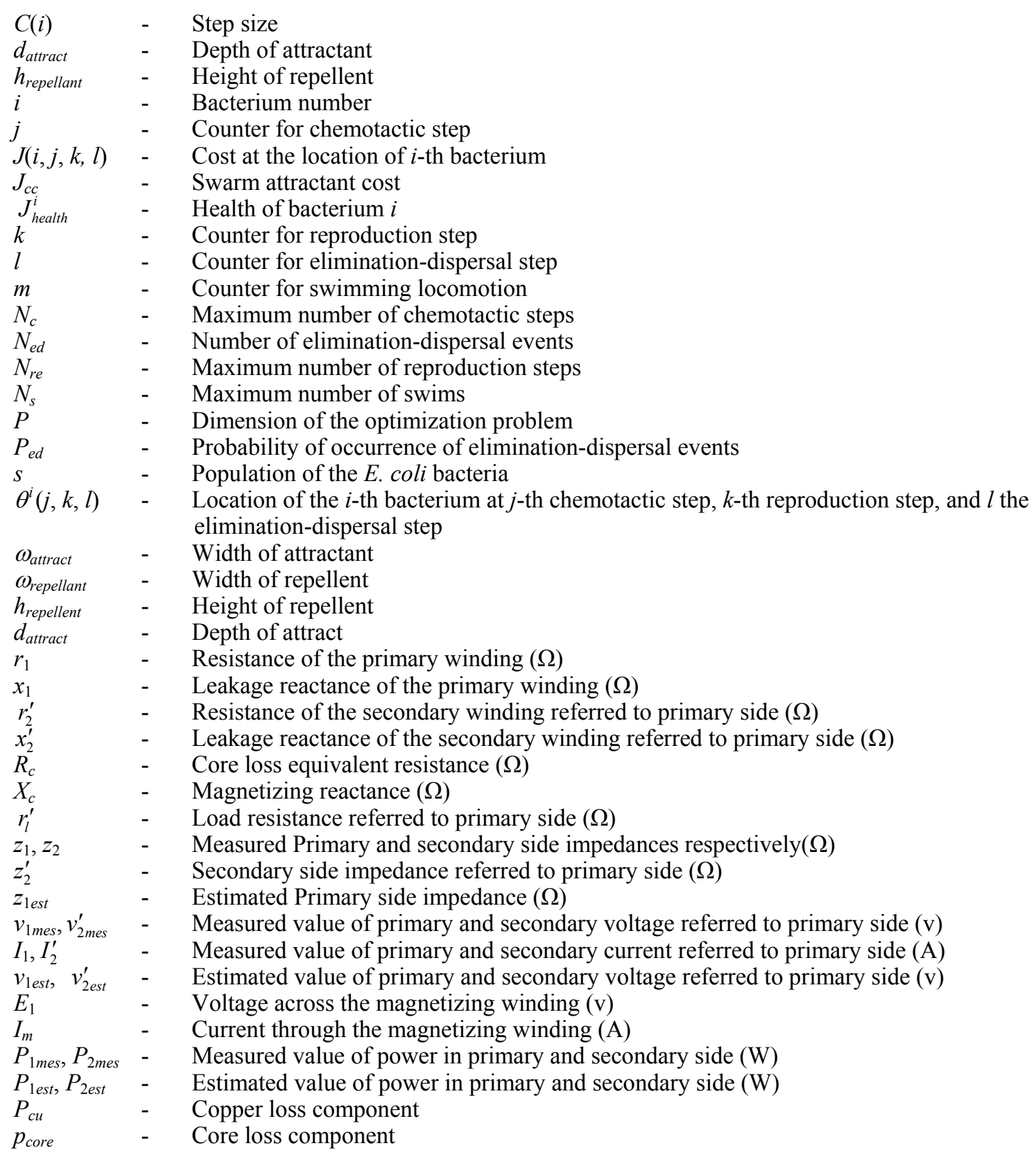

